\title{
Screening for Alcoholism Among Medical Inpatients: How Important Is Corroboration of Patient Self-Report?
}

\author{
Stephen T. Chermack, Kathleen Singer, and Thomas P. Beresford
}

\begin{abstract}
Little is known about the utility of collateral reports in substantiating self-report for individuals assessed in nonalcoholism treatment contexts. This study examined the concordance of 581 pairs of medical patient and collateral responses to a commonly used alcohol screening instrument, the CAGE Questions, as well as to reports of the patient's drinking consequences and alcohol consumption. Results demonstrated that patient/collateral concordance was marginal, but acceptable, on CAGE cut-off scores and, that similar to reports from alcoholism treatment settings, patients generally reported more drinking consequences than collaterals. Patient and collateral reports of the patient's alcohol consumption did not differ significantly. This pattem of patient and collateral reporting of alcohol consequences and consumption was found for both men and women, as well as for patients with a DSM-III-R diagnosis of alcohol dependence. The findings support the validity of patient self-report on alcoholism screening measures in medical settings. Furthermore, results demonstrated that the addition of collateral reports to information directly obtained from patients only modestly improved the identification of alcohol dependence. The overall findings indicate that alcohol screening can be done effectively and efficlently in medical settings.
\end{abstract}

Key Words: Acoholism, Screening, Validity, Self-report, Collateral.

$\mathbf{T}$ HERE IS ample evidence that individuals with alcohol dependence experience a variety of health problems and are frequent utilizers of medical services. It has been estimated that untreated alcoholics incur nearly double the health care costs of nonalcoholics. ${ }^{1}$ Routine screening for alcohol problems, followed by appropriate interventions, could markedly reduce the social and economic costs of alcohol problems. There is some evidence that treatment for alcohol problems reduces health care costs, primarily by reducing use of inpatient care. ${ }^{1}$ It is notable that, among individuals hospitalized for medical reasons, alcohol problems appear to be significantly more common than in the

From the John D. Dingell VA Medical Center (S.T.C.) and the Department of Psychiatry and Behavioral Neurosciences (S.T.C.), Wayne State University, Detroit, Michigan; the Department of Family Practice (KS.), University of Michigan Medical School, Ann Arbor, Michigan; and the University of Colorado Health Sciences Center (T.P.B.) and the VA Medical Center (T.P.B.), Denver, Colorado.

Received for publication August 22, 1997; accepted May 7, 1998

This study was supported by Grants ROI-AA-07236, P50-AA-07378, and $P-32-A A 07477$ from the National Institute on Alcohol Abuse and Alcoholism, and by Grant 003 from the Department of Veterans Affairs Alcohol Research Center (Denver, CO).

Reprint requests: Stephen T. Chermack, Ph.D., the John D. Dingell VA Medical Center, Psychiatry Service (116A), 4646 John R., Detroit, MI 48201.

Copyright $\mathbb{0} 1998$ by The Research Society on Alcoholism. general population. ${ }^{2}$ Thus, researchers have recommended the routine screening of hospital inpatients for alcohol problems. $^{3}$

There has been controversy about the validity of selfreport data for individuals with alcohol problems. It has been argued that such individuals may underreport alcohol consumption and drinking consequences because of denial of problems with alcohol. ${ }^{4}$ However, a number of investigators have noted several factors that may affect the validity of patient self-report, such as whether patients are sober when interviewed, characteristics of the patient and interviewer, the context in which individuals are assessed, ${ }^{5-7}$ and the type of data assessed (global vs. specific, subjective vs. objective, salient vs. insignificant). ${ }^{8}$ Recent studies have attempted to assess the validity of self-report data by examining the concordance of such information with information obtained from collateral sources, such as spouses, children, parents, or close friends who know the person well. ${ }^{7-12}$ These studies have demonstrated that, for alcoholics in treatment, substance abusers interviewed at follow-up from inpatient treatment, as well as individuals reporting the resolution of previous alcohol problems, self-report data in general appear to be consistent with collateral reports with regard to drinking habits and problems associated with alcohol use, and that the pattern of concordance does not appear to reflect denial or minimization. However, the concordance of patient and collateral reports for individuals not involved in treatment has received little study. Loethen and Khavari ${ }^{9}$ have suggested that the potential for underreporting alcohol problems is probably higher for alcoholics not in treatment, due to denial. It is not clear whether the concordance of patient and collateral reports is reduced by less reliable reporting among alcoholics not participating in treatment. If alcoholics not in treatment provide less reliable information or underreport alcohol problems, the effectiveness of screening measures could be reduced. On the other hand, if collateral information can be obtained in a cost-effective manner, this additional information may aid in identifying individuals with alcohol problems.

This study investigated the validity of patient self-reports among medical inpatients and focused on the potential utility of obtaining collateral information when screening for alcohol dependence among medical inpatients with the 
CAGE Questionnaire. ${ }^{13}$ The CAGE is a brief four-item self-report questionnaire asking about common correlates of heavy alcohol use, and it has been demonstrated to be a highly reliable and valid screening instrument for alcohol problems. ${ }^{13-16}$ For the collaterals in this study, the CAGE Questionnaire was modified to ask the collateral about the patient's alcohol use. A modified CAGE Questionnaire for collaterals as part of alcohol screening in medical settings is the most logical choice for attempting to corroborate patient self-report in a cost-effective manner due to its brevity and ease of administration.

Investigating the concordance of patient/collateral CAGE scores can provide information regarding the validity of patient self-report. However, the degree of concordance on the CAGE for patients and collaterals in inpatient medical settings has not been assessed. Concordance of patient and collateral CAGE reports would suggest that the patients are providing valid information. If the vast majority of hospitalized medical patients and collaterals are concordant on the CAGE, then obtaining further information from collateral informants may not be necessary to screen effectively for alcoholism.

Discordant responses, on the other hand, raise the possibility that either patients, collaterals, or both may not be reliable informants. Investigating the consistency of reporting of CAGE discordant patient/collateral pairs on other measures related to alcohol problems would provide information on the reliability of patient reports, and may suggest conditions in which additional collateral information can be of most utility. This also would address the potential impact of patient denial or minimization on alcohol screening. The primary threat to effective screening with the CAGE is when patients' scores are below cut-offs due to underreporting of alcohol consequences. As noted herein, underreporting of alcohol problems by alcoholics has been attributed to denial or minimization. ${ }^{9}$ One way of assessing the impact of such a response set is to examine reports of other problems associated with drinking for those discordant patient/collateral pairs in which the patient scored below the cut-off for alcohol problems. A pattern in which collaterals report significantly more problems would be expected if such patients adopted a response set consistent with denial/ minimization. Furthermore, the impact of such a response set also can be assessed by examining the consistency of patient/collateral reporting of alcohol problems and consumption levels for patients with a diagnosis of alcohol dependence. If denial or minimization is characteristic of alcohol-dependent individuals, then such patients would be expected to underreport alcohol problems and consumption relative to collaterals.

This study also examined the influence of patient and collateral reports in identifying patients with a diagnosis of alcohol dependence. The incremental validity of patient and collateral reports on the CAGE has not been investigated, and may provide important information regarding effective methods of screening for alcoholism. The goal of screening is to maximize accuracy and minimize the costs of obtaining relevant information. Because obtaining collateral information requires additional effort, time, and cost, it is important to assess whether the addition of collateral information to patient reports could enhance the effectiveness of alcohol screening procedures in medical settings.

\section{METHODS}

\section{Subjects}

Data for the present analysis were collected as part of a larger study designed to evaluate optimal screening methods for identifying covert alcoholism among medical/surgical patients. ${ }^{13}$ The larger study selected 1,262 participants at random, using random number tables, from daily admission lists of patients at the University of Michigan Hospitals during the period between January 1987 and April 1989. Of the 1,262 patients selected, 915 agreed to participate in the larger study. The present study examined a sample of approximately every second patient approached to participate in the larger study. These patients were asked by study personnel if a family member or close friend who knows them could be contacted as part of the study. Thus, of the $\sim 630$ potential participants for the corroboration aspect of the study, 581 individuals agreed to participate.

Of the 581 patients included in this analysis, the age of the participants ranged from 18 to 86 (mean $=48 ; S D=16.9$ ); $56 \%$ were men, and $89 \%$ were Caucasian, $10 \%$ African-American, and $2 \%$ other races. The majority of participants $(54 \%)$ had 12 years of education or less, and $59 \%$ of subjects had an annual income of less than $\$ 30,000$ (mean income range $=$ $\$ 15,000$ to $\$ 20,000$ ). Approximately $60 \%$ of participants were married and living with their spouses, with the remainder being single, divorced, separated, or widowed. According to a structured diagnostic interview, 178 (30.6\%) of the subjects met DSM-III-R criteria for alcohol dependence in their lifetime. Collaterals were $52 \%$ spouses/partners, $17 \%$ other relatives, $15 \%$ parents, $11 \%$ siblings, and $5 \%$ friends. The majority of collaterals $(87 \%)$ reported that they had known the patient for $>10$ years, $83 \%$ indicated that they had contact with the patient at least several times a week.

\section{Procedures}

Following informed consent, patients were interviewed using a semistructured interview schedule incorporating Vaillant's Interview Schedule for Alcohol Use, ${ }^{17}$ and several standard alcoholism screening measures, including the CAGE Questionnaire ${ }^{15}$ and the MAST ${ }^{18}$ The interview required $\sim 1 \mathrm{hr}$ for participants to complete. The structured interview contained a number of questions reflecting whether participants met each of the nine DSM-III-R criteria for alcohol dependence. From this interview, it was possibie to ascertain whether participants met DSM-III-R criteria for lifetime alcohol dependence. The CAGE Questionnaire asks respondents to answer either yes or no to the following four questions: Have you ever felt you should Cut down on your drinking? Have other people Annoyed you by criticizing your drinking? Have you ever felt Guilty about drinking? Have you ever taken a drink in the morning to steady your nerves or get rid of a hangover? (Eyeopener).

Patients also provided the name of a collateral and consent for study personnel to interview this person. The identified collateral was contacted by telephone generally within 2 weeks and was interviewed using a brief (20-min) structured interview constructed to corroborate alcohol consumption and drinking consequences reported by the patient. The structured interview consisted of modifications of the CAGE Questionnaire, a subset of MAST items, and questions regarding the patient's alcohol consumption and withdrawal symptoms. The modifications involved phrasing the items to ask the collateral about the patient's alcohol use (e.g., Does he/she ever have a drink first thing in the morning to steady his/her nerves or get rid of a hangover? Has he/she ever gotten into 
Table 1. No. of Reported Drinking Consequences for CAGE Concordant and Discordant Patient/Collateral Pairs

\begin{tabular}{|c|c|c|c|c|c|c|c|}
\hline \multirow[b]{2}{*}{ Group } & \multirow{2}{*}{$\begin{array}{c}\text { No. of } \\
\text { pairs }\end{array}$} & \multicolumn{2}{|c|}{ Patients' reports } & \multicolumn{2}{|c|}{ Collaterals' reports } & \multirow[b]{2}{*}{$t$ value } & \multirow[b]{2}{*}{$r$} \\
\hline & & Mean (SD) & Range & Mean (SD) & Range & & \\
\hline Total sample & 581 & $2.85(3.34)$ & $0-19$ & $1.55(2.86)$ & $0-18$ & $10.59^{*}$ & $0.564^{*}$ \\
\hline Negative patient/negative collateral & 388 & $1.40(1.87)$ & $0-10$ & $0.55(1.09)$ & $0-10$ & $8.37^{*}$ & $0.174^{*}$ \\
\hline Positive patient/positive collateral & 66 & $7.53(4.23)$ & $0-19$ & $6.85(4.45)$ & $0-18$ & 1.27 & $0.496^{*}$ \\
\hline Positive patient/negative collateral & 98 & $5.30(3.30)$ & $0-19$ & $1.38(1.77)$ & $0-8$ & $12.07^{*}$ & $0.316^{*}$ \\
\hline Negative patient/positive collateral & 29 & $3.45(2.89)$ & $0-12$ & $3.59(3.45)$ & $0-14$ & -0.18 & 0.195 \\
\hline
\end{tabular}

- $p<0.001$.

trouble at work because of drinking? How much does he/she usually drink per occasion?).

\section{Data Analysis}

CAGE Questionnaire responses for both patients and collaterals were dichotomized to reflect whether the score was above or below the standard cut-off of 2 affirmative answers as indicative of alcohol problems. Cut-off scores were used so that analyses would focus on the manner in which the CAGE is typically used in clinical practice. The concordance of patient and collateral CAGE cut-off scores was then assessed with the Yule's $Y$ statistic. Recent studies have suggested that Yule's $Y$ is the statistic of choice to assess concordance of patient-collateral reports and that a value of 0.50 or above is indicative of "acceptable" concordance. ${ }^{8}$

To investigate whether concordance and discordance on the CAGE questionnaire was related to the consistency of reporting on other indicators of alcohol problems, the following four groups were constructed based on the pattern of patient and collateral CAGE scores: (1) negative patient/negative collateral (both patient and collateral score below the cut-off for alcohol problems); (2) positive patient/positive collateral (both patient and collateral score above the cut-off for alcohol problems); (3) positive patient/negative collateral (only the patient's score above the cut-off); and (4) negative patient/positive collateral (only the collateral's score above the cut-off). A summary score indicating the patient's total number of alcohol consequences was constructed for both patient and collateral reports. This sum consisted of 22 items that were administered to both the patient and the collateral, and included items from the MAST (e.g., regarding history of AA attendance, disruptions in social relationships and occupational functioning, history of blackouts, positive treatment history), as well as questions regarding withdrawal symptoms following drinking episodes (e.g., shakes, headaches, flu-like symptoms, seizures, hallucinations, DTs). Paired samples $t$ tests were performed to assess whether patient/collateral pairs in each group differed in the reported number of alcohol consequences, as well as measures of reported alcohol consumption. Bivariate correlations were computed to assess the association between patient and collateral reports on these consequences. To assess the validity of self-report information for individuals with alcohol problems, paired samples $t$ tests and bivariate correlations were also performed for patient/collateral pairs in which the patient was positive for a DSM-III-R diagnosis of alcohol dependence.

Hierarchical logistic regression was used to assess the contributions of demographic variables, patient CAGE cut-off score, and collateral CAGE cut-off score in predicting alcohol dependence. The variables included at each step was based on trying to obtain the most accurate classification model, while considering the efficiency with which relevant screening information could be obtained in practice. For this analysis, demographic information was entered at stage 1 to adjust for potential demographic influences. Patients' reported CAGE cut-off score was entered at stage 2, and collaterals' CAGE score was entered at stage 3 . The rationale of the analytic strategy was that it would provide information regarding whether the addition of the collateral CAGE to the standard CAGE enhances the identification of alcohol dependence. This method of analysis also provides information about the contribution of standard screening methods in predicting alcohol dependence.
RESULTS

\section{Concordance of Patient and Collateral CAGE Cut-off Scores}

An analysis of patient and collateral CAGE scores revealed "acceptable," but somewhat marginal concordance $(\mathrm{Y}=0.50)$. On the basis of standard CAGE cut-off scores (a score of 2 or more is suggestive of alcohol dependence), $66.8 \%$ of patient/collateral pairs provided concordant responses below cut-off scores (negative patient/negative collateral), and $11.4 \%$ of patient/collateral pairs provided concordant responses above cut-off scores (positive patient/ positive collateral). Thus, $\sim 21.9 \%$ of the patient/collateral pairs were discordant on the CAGE. Seventy-seven percent of the discordant pairs had only the patient score above the cut-off (positive patient/negative collateral), and $22.9 \%$ had only collaterals providing a score above the cut-off (negative patient/positive collateral). Thus, the negative patient/ positive collateral pattern of CAGE responding accounted for just $5 \%$ of the total patient/collateral pairs. The four groups with different patterns of CAGE concordance differed in gender composition, $\chi^{2}(3 d f)=43.67, p<0.001$. The percentage of male subjects was $47 \%$ for the negative/ negative group, $86 \%$ for the positive/positive group, $63 \%$ for the positive/negative group, and $76 \%$ for the negative/ positive group.

\section{Drinking Consequences and Consumption Levels for Patient/Collateral Pairs}

Analyses of the number of drinking consequences for patient and collateral reports are presented in Table 1. For the total sample, patients reported significantly more alcohol consequences than collaterals, and patient and collateral reports were significantly correlated. To investigate whether the patterns of patient and collateral reporting on the CAGE were similar for other indicators of alcohol problems, additional analyses examined patient and collateral reporting for each of four groups defined by their concordance or discordance on the CAGE Questionnaire. The results indicated that patient and collateral reports were significantly, albeit marginally, correlated, with the exception of the negative patient/positive collateral group. The results also demonstrated that, among negative patient/negative collateral and positive patient/negative collateral pairs, patients reported significantly more alcohol 
Table 2. No. of Reported Drinks/Week for CAGE Concordant and Discordant Patient/Collateral Pairs

\begin{tabular}{|c|c|c|c|c|c|c|c|}
\hline \multirow[b]{2}{*}{ Group } & \multirow{2}{*}{$\begin{array}{c}\text { No. of } \\
\text { pairs }\end{array}$} & \multicolumn{2}{|c|}{ Patients' reports } & \multicolumn{2}{|c|}{ Collaterals' reports } & \multirow[b]{2}{*}{$t$ value } & \multirow[b]{2}{*}{$r$} \\
\hline & & Mean (SD) & Range & Mean (SD) & Range & & \\
\hline Total sample & 581 & $3.47(9.68)$ & $0-75$ & $3.21(10.34)$ & $0-70$ & 0.71 & $0.623^{*}$ \\
\hline Negative patient/negative collateral & 388 & $1.84(5.35)$ & $0-52$ & $1.37(3.91)$ & $0-24$ & 2.17 & $0.609^{*}$ \\
\hline Positive patient/positive collateral & 66 & $8.24(16.17)$ & $0-75$ & $11.62(22.68)$ & $0-70$ & -1.44 & $0.565^{\star}$ \\
\hline Positive patient/negative collateral & 98 & $4.99(10.37)$ & $0-52$ & $2.63(6.89)$ & $0-56$ & 2.47 & $0.461^{*}$ \\
\hline Negative patient/positive collateral & 29 & $9.37(20.73)$ & $0-75$ & $10.79(19.22)$ & $0-70$ & -0.80 & $0.852^{*}$ \\
\hline
\end{tabular}

$* p<0.001$.

problems than collaterals. The positive patient/positive collateral and negative patient/positive collateral pairs did not differ in the number of consequences.

Additional analyses revealed that the pattern of patient and collateral reporting of alcohol consequences was consistent for both male and female patients. Both male and female patients reported significantly more alcohol consequences than collaterals, and their reports were significantly correlated with collateral reports. The analyses of drinking consequences provided no evidence that patients underreport alcohol consequences, compared with collateral sources. In fact, these results suggest that, when differences occur, it appears to be due to patients reporting more problems than collaterals.

Table 2 contains analyses of patient and collateral reports of the mean number of drinks consumed per week. Neither the total sample pairs nor the two concordant and two discordant CAGE group pairs differed significantly in reports of the mean number of drinks consumed per week. In addition, patient and collateral reports were significantly correlated for all groups of patient/collateral pairs. Additional analyses revealed this same pattern of patient and collateral reporting for both men and women. There was no evidence that patients underreport their levels of alcohol consumption when compared with the reports of collateral informants.

\section{Alcohol-Dependent Patients}

The analyses described herein demonstrated that medical patients in general do not appear to underreport indicators of alcohol problems relative to collaterals, whether or not their scores on a brief alcoholism screening test are concordant with collaterals. However, of most concern to clinicians is the reliability and validity of self-report information for patients with significant alcohol problems. Analyses of patient/collateral pairs in which the patient met DSM-III-R criteria for alcohol dependence were similar to the results for medical patients in general. Alcoholdependent patients reported significantly more consequences than did collaterals $[t(177)=11.46, p<0.001]$, and patient and collateral reports were significantly correlated $(r=0.523, p<0.001)$. This pattern of results was found for both men and women patients. The mean number of consequences reported by patients and collaterals were $6.48(\mathrm{SD}=3.60)$ and $3.27(\mathrm{SD}=4.03)$, respectively. Patients and collaterals did not differ significantly on re-
Table 3. Hierarchical Logistic Regression Predicting Alcohol Dependence

\begin{tabular}{|c|c|c|c|}
\hline & $\begin{array}{c}\text { Stage } 1 \\
\text { Odds ratio }\end{array}$ & $\begin{array}{c}\text { Stage } 2 \\
\text { Odds ratio }\end{array}$ & $\begin{array}{c}\text { Stage } 3 \\
\text { Odds ratio }\end{array}$ \\
\hline \multicolumn{4}{|l|}{ Demographic variables } \\
\hline Age & $0.90^{\star *}$ & 0.96 & 0.98 \\
\hline Marital status & $1.05^{\star}$ & 1.00 & 1.00 \\
\hline Race & 1.00 & 1.00 & 1.00 \\
\hline Income & 1.00 & 1.00 & 1.00 \\
\hline Gender & $1.24^{* *}$ & $1.13^{* *}$ & $1.12^{* *}$ \\
\hline Patient CAGE cut-off score & & $1.63^{* \star}$ & $1.74^{* * *}$ \\
\hline Collateral CAGE cut-off score & & & $1.16^{\star *}$ \\
\hline Model improvement & $75.11^{\star \star}$ & $205.72^{* *}$ & $10.75^{* *}$ \\
\hline$d f$ & 21 & 1 & 1 \\
\hline Goodness-of-fit & 529.47 & 501.36 & 523.25 \\
\hline Sensitivity (\%) & 30.11 & 72.73 & 73.30 \\
\hline Specificity (\%) & 89.89 & 92.62 & 92.90 \\
\hline$\%$ Correct & 70.48 & 86.16 & 86.53 \\
\hline
\end{tabular}

${ }^{\star} p<0.05 ; " * p<0.01$.

ported number of drinks per week $[t(177)=0.13]$ and their reports were correlated at the 0.001 level $(r=0.437)$. The mean number of drinks per week reported by patients and collaterals were $5.76(\mathrm{SD}=11.62)$ and $5.62(\mathrm{SD}=14.83)$, respectively. The same pattern of results were observed for both male and female alcohol-dependent patients. Furthermore, the same pattern of results were observed when alcohol-dependent patients reporting 3 months or more of abstinence were excluded from the analysis. Specifically, patients reported significantly more alcohol consequences than collaterals $[t(110)=9.58, p<0.001]$, and patients' and collaterals' reports of the patients' alcohol consumption did not differ significantly $[t(110)=1.04]$. The mean number of drinks per week reported by patients and collaterals for patients drinking within the past 3 months were 9.32 ( $\mathrm{SD}=$ $13.63)$ and $7.79(\mathrm{SD}=16.05)$, respectively. These results indicated that patients with significant alcohol problems did not underreport alcohol problems or consumption levels relative to collateral respondents.

\section{Multivariate Analyses of Alcohol Dependence}

The results of the hierarchical logistic regression analysis in classifying alcohol dependence is displayed in Table 3. At stage 1 , age, marital status, and gender were significantly related to having a diagnosis of alcohol dependence. The fit of the model significantly improved with the inclusion of the patient CAGE cut-off score at stage 2. Furthermore, 
the fit of the model also significantly improved with the inclusion of the collateral CAGE cut-off score at stage 3 . For the final model, patient-reported CAGE cut-off scores, patient gender (male), and collateral CAGE score were significantly predictive of a diagnosis of alcohol dependence. In terms of the accuracy of the model in classifying alcohol dependence, it is apparent that the patients' CAGE score had adequate sensitivity, specificity, and correctly classified the majority of patients, and that the addition of the collateral CAGE resulted in only a very modest increase in classification accuracy.

\section{DISCUSSION}

Our results demonstrate that the concordance of patient and collateral reports for one major alcoholism screening measure, the CAGE, is marginal. Approximately $23 \%$ of the patient/collateral pairs were discordant. However, it was a minority of the discordant pairs on the CAGE (29/ $127)$ in which only the collateral scored above the cut-off, and this pattern accounted for just $5 \%$ of the total number of patient/collateral pairs. Additional analyses revealed that discordant responding on the CAGE questionnaire did not appear to reflect a tendency toward denial or minimization on other questions assessing alcohol consequences and consumption. Furthermore, analyses of drinking consequences demonstrated that, similar to reports from treatment settings, ${ }^{9}$ patients tended to report either more negative drinking consequences than collaterals, or did not markedly differ in reporting of drinking consequences. $\mathrm{Pa}$ tients and collaterals also did not differ significantly in their reports of the patient's alcohol consumption. The pattern of findings for patients with diagnoses of alcohol dependence was similar to those of the total patient sample. In addition, there was no evidence that the pattern of patient and collateral reporting on measures of alcohol consequences and consumption differed for male and female patients. The findings of this study suggest that medical patients, alcohol-dependent or not, provide valid information on standard alcohol screening questions.

The results of the logistic regression analysis generally were consistent with this perspective. The inclusion of a collateral CAGE only modestly improved the accuracy of screening beyond the CAGE score obtained directly from patients. These findings verified that alcohol screening in medical settings can be done effectively, based solely on patient reports, and suggest that the use of staff time and resources to obtain collateral reports are unwarranted by the modest increase in the accuracy of screening. The results are consistent with the results of other studies, demonstrating that alcohol problems can be screened effectively in medical settings. ${ }^{3,13,16,19}$ Given the evidence that individuals with alcohol problems can benefit from a variety of low cost interventions aimed at problematic alcohol use,,$^{20,21}$ the finding that patients can be screened effectively with brief screening instruments should be encouraging to health care professionals.

The results of this study should be interpreted in the proper context. The procedures used to interview patients may have influenced the validity of their self-report. It has been posited that factors such as the characteristics of the patient and interviewer, type of data assessed, and the context of the interview are factors that may impact the validity of patient self-report. ${ }^{7}$ In the present study, interviewers all wore white laboratory coats and introduced themselves as research assistants. Questions regarding alcohol problems and consumption patterns were asked in a direct, but nonconfrontational manner, and the inpatient context of the assessment was clearly associated with the patients' medical health care. It is possible that these factors enhanced the validity of patient reporting. It should be noted that these procedures would not be difficult to implement in medical settings and would likely maximize the validity of information obtained from patients. A variety of medical staff members-including medical assistants, nurses, and physicians-could be trained to administer alcohol screening questions. It is likely that such health care providers would also obtain valid information from patients given the health care context of the screening questions and the credibility of being a member of a hospital staff. However, given limited progress in educating medical personal to administer alcohol screening instruments ${ }^{22,23}$ research is needed to identify and overcome barriers to underutilization of screening by medical personnel, or to examine the cost-effectiveness of using other staff (alcohol specialists, social workers) to handle alcoholism screening, brief intervention, and referral issues.

Although the results of this study suggest that obtaining collateral information may only modestly improve screening for alcohol problems, it is likely that there are some situations in which obtaining collateral information would be particularly important. For example, it is possible that collateral reports may aid in identifying alcohol problems for patients who are unable to respond to questions in a valid manner due to medical conditions, injuries, or cognitive limitations. It could also be argued that obtaining collateral information would be the only means of identifying patients with alcohol problems for those patients who refuse to answer screening questions about their alcohol use. However, in practice, it would be difficult to persuade such individuals to agree to having a collateral provide information about their alcohol use, and such individuals would not be likely to consider a subsequent intervention focusing on their alcohol consumption. Thus, there would probably be little benefit from pursuing collateral information in such circumstances.

This study provides evidence that the effectiveness of screening for alcohol problems in medical settings is not compromised by poor validity of patient reports. Furthermore, the results revealed that additional information obtained from collaterals would not substantially enhance the 
effectiveness of alcohol screening in the vast majority of medical patients. Nevertheless, the methodology used in this study has some limitations. First, although the study used a comprehensive structured interview assessing alcohol-related consequences and symptoms of dependence to establish whether participants met lifetime DSMIII-R diagnostic criteria, there are no current psychometric data regarding the reliability and validity of this interview. Other structured interviews, such as the Diagnostic Interview Schedule (DIS) ${ }^{24}$ or the Structured Clinical Interview for DSM-III-R (SCID) ${ }^{25}$ have had a more thorough examination of their reliability and validity. These instruments could provide more detailed information regarding both current and lifetime alcohol symptoms. Second, the study focused on DSM-III-R rather than DSM-IV criteria. Third, despite its efficiency, there are some inherent limitations of the CAGE items in that the items assess lifetime history and do not consider whether or not individuals are currently drinking. This could increase the false-positive rate in screening due to individuals whose past alcohol-related problems have resolved. Further research could address the limitations of this study by focusing on other screening techniques that incorporate questions about current drinking (e.g., the AUDIT), and by using alternative wellvalidated methods of determining both lifetime and current alcohol-related diagnoses (e.g., DIS or SCID), and by using current diagnostic criteria. Finally, additional research is needed to assess the validity of patient self-report on screening measures in today's more short-term and outpatient based health care delivery system, as well as other settings. For example, it is important to verify the validity of self-report on alcohol screening instruments for primary care, community, psychiatric, and forensic settings, and it is not known whether the addition of collateral information would enhance screening in such settings.

\section{REFERENCES}

1. Holder HD: Alcoholism treatment and potential health care cost saving. Med Care 25:52-71, 1987

2. Moore RD, Bone LR, Geller G, Mamon JA, Stokes EJ, Levine DM: Prevalence, detection and treatment of alcoholism in hospitalized patients. JAMA 261:403-407, 1989

3. Umbricht-Schneiter A, Santora P, Moore RD: Alcohol abuse: Comparison of two methods for assessing its prevalence and associated morbidity in hospitalized patients. Am J Med 91:110-118, 1991

4. Watson CG, Tilleskjor C, Hoodecheck-Schow EA, Pucel J, Jacobs L: Do alcoholics give valid self-reports. J Stud Alcohol 45:344-348, 1984

5. Babor TF, Stephens RS, Marlatt GA: Verbal report methods in clinical research on alcoholism: Response bias and its minimization. J Stud Alcohol 48:410-424, 1987

6. Midanik LT: Validity of self-reported alcohol use: A literature review and assessment. Br J Addict 83:1019-1029, 1988

7. Tucker JA, Vuchinich RE, Harris CV, Govornki MG, Rudd EJ: Agreement between subject and collateral verbal reports of alcohol consumption in older adults. J Stud Alcohol 52:148-155, 1991

8. Hoffmann NG, Ninonuevo FG: Concurrent validation of substance abusers self-reports against collateral information: Percentage agreement vs. K vs. Yule's Y. Alcohol Clin Exp Res 18:231-237

9. Loethen GJ, Khavari KA: Comparison of the self-administered alcoholism screening test (SAAST) and the Khavari Alcohol Test (KAT): Results from an alcoholic population and their collaterals. Alcohol Clin Exp Res 14:756-760, 1990

10. Wolber G, Carne WF, Alexander R: The validity of self-reported abstinence and quality sobriety following chemical dependency treatment. Int J Addict 25:495-513, 1990

11. Maisto SA, Sobell LC, Sobell MB: Reliability of self-reports of low ethanol consumption by problem drinkers over 18 months of follow-up. Drug Alcohol Depend 9:273-278, 1982

12. Sobell LC, Cunningham JA, Sobell MB, Toneatto T: A life-span perspective on natural recovery (self-change) from alcohol problems, in Baer JS, Marlatt GA, McMahon RJ (eds): Addictive Behaviors Across the Life Span. Newberry Park, Sage, 1993, p 34

13. Ewing JA: Detecting alcoholism, the CAGE questionnaire. JAMA 252:1905-1907, 1984

14. Beresford TP, Blow FC, Hill E, Singer K, Lucey MR: Comparison of CAGE questionnaire and computer-assisted laboratory profiles in screening for covert alcoholism. Lancet 336:482-485, 1990

15. Buchsbaum DG, Buchanan RG, Welsh J, Centor RM, Schnoll SH: Screening for drinking disorders in the elderly using the CAGE questionnaire. J Am Geriatr Soc 40:662-665, 1992

16. McIntosh MC, Leight G, Baldwin NJ: Using the CAGE and measures of alcohol consumption in family practice. Can Fam Physician 40:1546-1553, 1994

17. Vaillant GE: The Natural History of Alcoholism. Cambridge, MA, Harvard University Press, 1983

18. Selzer ML: The Michigan Alcoholism Screening Test: The quest for a new diagnostic instrument. Am J Psychiatry 127:1653-1658, 1971

19. Morton JL, Manganaro MA, Jones TV: Performance of alcoholism screening questionnaires in elderly veterans. Am J Med 101:153-159, 1997

20. Babor TF: Avoiding the horrid and beastly sin of drunkenness: Does dissuasion make a difference. J Consult Clin Psychol 62:1116-1126, 1996

21. Fleming MR, Barry KL, Manwell LB, Johnson K, London R: Brief physician advice for problem alcohol drinkers: A randomized controlled trial in community-based primary care practices. JAMA 277:1039-1045, 1997

22. Wenrich MD, Paauw DS, Carline JD, Curtis JR, Ramsey PG: Do primary care physicians screen patients about alcohol intake using the CAGE Questions? J Gen Intern Med 10:631-634, 1995

23. McCrady BS, Slade J, Richter SS, Pfeifer C, Morgan TJ: Involving health care workers in screening for alcohol problems. J Addict Dis $15: 45-58,1996$

24. Robins LN, Helzer JE, Croughan J, Ratcliff KS: National Institute of Mental Health Diagnostic Interview Schedule. Arch Gen Psychiatry 38:381-389, 1981

25. Spitzer RL, Williams JBW, Gibbon M, First MB: User's Guide for the Structured Clinical Interview for DSM-III-R. Washington, D.C.: American Psychiatric Association Press, 1990 